\title{
Pseudostate description of breakup in coupled-reaction-channel method: Numerical study of nonorthogonality effects
}

\author{
Zeki C. Kuruoğlu \\ Department of Chemistry, Bilkent University, Ankara, Turkey
}

(Received 17 July 1990)

\begin{abstract}
To simulate the effects of the breakup channel on rearrangement amplitudes, the conventional coupled-reaction-channel (CRC) expansion is augmented by pseudoreaction channels. The construction of the projector for the extended CRC space is discussed, and transition-operator equations on this space are given. By solving the full and post-approximation forms of the CRC equations for a model three-particle problem, the crucial role played by the nonorthogonality terms is demonstrated.
\end{abstract}

\section{INTRODUCTION}

The conventional coupled-reaction-channel method ${ }^{1,2}$ is a versatile and efficient method in treating rearrangement scattering, as, e.g., the recent successful applications $^{3}$ to reactive atom-diatom collisions amply demonstrate. The efficiency of the CRC idea has also been borne out in recent time-dependent wave-packet treatments ${ }^{4,5}$ of three-body dynamics. A better understanding of the theoretical underpinnings of the CRC ansatz has emerged through the works of, e.g., Chandler and Gibson, ${ }^{6}$ Bencze, Chandler, and Gibson, ${ }^{7}$ and Birse and Redish. ${ }^{8}$ In particular, the troublesome possibility of overcompleteness in the CRC ansatz has been shown to be curable by the use of generalized-inverse methods. ${ }^{9}$ This paves the way to generalize the CRC method to include the breakup channels on equal footing with the two-fragment rearrangements. Such a generalized CRC ansatz has recently been used by the present author $^{5}$ to solve the time-dependent Schrödinger equation for a three-particle problem at energies above the breakup threshold.

Whether in time-independent (stationary) or timedependent contexts, the success of the CRC method stems from the particular construction of the approximation space. The CRC approximation space is a union of the subspaces for a chosen set of arrangements. In standard applications, only two-fragment rearrangement channels are included in the construction of the CRC approximation space. Note that subspaces for different arrangements are not orthogonal, and the success of the CRC expansion ansatz is crucially dependent on the exact inclusion of the non-orthogonality kernel. Since in most previous applications ${ }^{10}$ of the CRC method in nuclear physics, the non-orthogonality effects had been ignored, it is the purpose of this article to show the importance of non-orthogonality effects on a numerically solvable model. The same question had been considered by Vincent and Cotanch ${ }^{2}$ formally and semiquantitatively for systems involving only two rearrangement channels. Our model involves the breakup channel as well as three rearrangement channels.

The formally-correct inclusion of the breakup channel into the CRC ansatz can be done within the ChandlerGibson approach, ${ }^{6}$ in which the two-fragment subspaces are to be augmented by a breakup subspace. For the breakup boundary conditions to be compatible with the expansion ansatz, the projector onto the breakup subspace has to commute with the asymptotic Hamiltonian for the breakup channel. This condition can be met by constructing the breakup subspace using a finite set of functions in hyperangular variables. Such an approach has been used by the present author in time-dependent wave-packet calculations, ${ }^{5}$ and the time-independent version is currently under study.

Another more ad hoc approach to handle the breakup channel is to augment ${ }^{11}$ the conventional CRC expansion (involving only the asymptotic rearrangement channels) with pseudorearrangement states (or channels). The pseudostates in question are square-integrable states in the continuum obtained by diagonalizing the (internal parts of the) rearrangement-channel Hamiltonians in finite approximation subspaces (in internal variables) for each rearrangement. In effect, the rearrangementchannel Hamiltonians (excluding the kinetic-energy operator for the relative motion of two fragments) are replaced by their restrictions on finite approximation subspaces. Of course, the full breakup-channel Hamiltonian does not commute with the projectors of the supspaces spanned by such pseudostates. That is, the correct breakup boundary conditions cannot be accommodated within this approximation space, and are replaced by approximate ones appropriate for two-fragment channels. Although the theoretical basis and justification of this approach is not well understood, numerical studies ${ }^{11}$ indicate that it is a convergeable procedure as far as rearrangement amplitudes are concerned. Pseudoreaction channels with two-fragment-type boundary conditions simulate the effect of the breakup channel on the reaction amplitudes by providing an outlet for the asymptotic breakup flux.

Note that the pseudostate description of the breakup 
channel within the time-dependent wave-packet approach is more natural since all we need is a sufficiently large $L^{2}$ approximation space. In fact, the expansion ansatz used by Kuruoglu and Levin ${ }^{4}$ in time-dependent wave-packet calculations of a three-particle problem is equivalent to a pseudostate-augmented CRC expansion.

\section{CONSTRUCTION OF THE CRC APPROXIMATION SPACE}

The partition $(\alpha)(\beta \gamma)$ of the three particles will be referred to as the $\alpha$ th rearrangement, whose Jacobi momenta are denoted by $\mathbf{p}_{\alpha}$ and $\mathbf{q}_{\alpha}$, with the corresponding reduced masses being $\mu_{\alpha}$ and $\nu_{\alpha}$, respectively. The kinetic-energy operator $H_{0}$ can be written as $H_{0}=k_{\alpha}+K_{\alpha}$, where $k_{\alpha}=p_{\alpha}^{2} /\left(2 \mu_{\alpha}\right)$, and $K_{\alpha}=$ $q_{\alpha}^{2} /\left(2 \nu_{\alpha}\right), \alpha=1,2,3$. The internal Hamiltonian for the pair $(\beta \gamma)$ is $h_{\alpha}=k_{\alpha}+V_{\alpha}$, where $V_{\alpha}$ is the potential between particles $\beta$ and $\gamma$. Bound states of $h_{\alpha}$ are denoted $\left|\varphi_{\alpha n}\right\rangle, n=1, \ldots, N_{\alpha}^{\text {bnd }}$ with energies $\epsilon_{\alpha n}$. The rearrangement-channel Hamiltonian $H_{\alpha}$ ( $\equiv K_{\alpha}+h_{\alpha}$ ) have the eigenkets $\left|\varphi_{\alpha n} \mathbf{q}_{\alpha}\right\rangle$ with energies $E_{\alpha n q}=\epsilon_{\alpha n}+q_{\alpha}^{2} /\left(2 \nu_{\alpha}\right)$.

To construct the approximation subspace for the $\alpha$ th rearrangement, we take a suitable orthonormal set of $L^{2}$ basis functions $u_{\alpha n}\left(\mathbf{p}_{\alpha}\right), n=1,2, \ldots, M_{\alpha}$. By diagonalizing the pair Hamiltonian $h_{\alpha}$ in this subspace, a set of pseudo-states $\left\{\left|\phi_{\alpha n}\right\rangle\right\}_{n=1}^{n=M_{\alpha}}$ are constructed: $\left\langle\phi_{\alpha m}\left|h_{\alpha}\right| \phi_{\alpha n}\right\rangle=\tilde{\epsilon}_{\alpha n} \delta_{n m}$, where $\left|\phi_{\alpha n}\right\rangle=$ $\sum_{n^{\prime}=1}^{M_{\alpha}}\left|u_{\alpha n^{\prime}}\right\rangle c_{\alpha, n^{\prime} n}$. The expansion coefficients are de- termined by the eigenvalue problem $\mathbf{h}_{\alpha} \mathbf{c}_{\alpha, n}=\tilde{\epsilon}_{\alpha n} \mathbf{c}_{\alpha, n}$, where $\left(\mathbf{h}_{\alpha}\right)_{n^{\prime} n}=\left\langle u_{\alpha n^{\prime}}\left|h_{\alpha}\right| u_{\alpha n}\right\rangle$ and $\mathbf{c}_{\alpha, n}$ is the column of expansion coefficients $c_{\alpha, n^{\prime} n}$. The basis size $M_{\alpha}$ ( $\gg N_{\alpha}^{\text {bnd }}$ ) is chosen such that the first $N_{\alpha}^{\text {bnd }}$ pseudostates are good approximations to the exact bound states, i.e., $\tilde{\epsilon}_{\alpha n} \approx \epsilon_{\alpha n}$, and $\left|\phi_{\alpha n}\right\rangle \approx\left|\varphi_{\alpha n}\right\rangle, n=1, \ldots, N_{\alpha}^{\text {bnd }}$. Using the first $N_{\alpha}$ pseudo states $\left(N_{\alpha}^{\text {bnd }}<N_{\alpha} \leq M_{\alpha}\right.$ ), the projector $\Pi_{\alpha}$ characterizing the approximation subspace for the rearrangement $(\alpha)(\beta \gamma)$ is now given as

$$
\Pi_{\alpha}=\sum_{n=1}^{N_{\alpha}} \int \mathrm{d} \mathbf{q}_{\alpha}\left|\phi_{\alpha n} \mathbf{q}_{\alpha}\right\rangle\left\langle\phi_{\alpha n} \mathbf{q}_{\alpha}\right|
$$

The full CRC approximation space is then taken as the union of three rearrangement subspaces. Since the subspaces for two distinct arrangements are not orthogonal (i.e., $\Pi_{\alpha} \Pi_{\beta} \neq \delta_{\alpha \beta} \Pi_{\alpha}$ for $\alpha \neq \beta$ ), the approximation space is not a simple direct sum of these subspaces. If rearrangement bases are each pushed to completeness, overcompleteness problems would arise. In practice, the linear dependence can usually be avoided by working with finite bases. If formal or numerical linear dependence arises, appropriate pseudo-inverse techniques have to be employed.

To construct the projector $\Pi^{\mathrm{CRC}}$ on the full CRC approximation space, we need the inverse of the overlap kernel $\Delta_{\alpha n, \beta n^{\prime}}\left(\mathbf{q}_{\alpha}, \mathbf{q}_{\beta}\right) \equiv\left\langle\phi_{\alpha n} \mathbf{q}_{\alpha} \mid \phi_{\beta n^{\prime}} \mathbf{q}_{\beta}\right\rangle$. If there is no linear dependence, then the inverse $\Lambda_{\alpha n, \beta n^{\prime}}\left(\mathbf{q}_{\alpha}, \mathbf{q}_{\beta}\right)$ of the overlap kernel (in abstract notation $\Lambda \equiv \Delta^{-1}$ ) can be obtained by solving

$$
\Lambda_{\alpha n, \beta n^{\prime}}\left(\mathbf{q}_{\alpha}, \mathbf{q}_{\beta}\right)=\delta_{\alpha \beta} \delta_{n n^{\prime}} \delta\left(\mathbf{q}_{\alpha}-\mathbf{q}_{\beta}\right)-\sum_{\gamma=1}^{3} \sum_{n^{\prime \prime}=1}^{N_{\gamma}} \int \mathrm{d} \mathbf{q}_{\gamma} \bar{\delta}_{\alpha \gamma} \Delta_{\alpha n, \gamma n^{\prime \prime}}\left(\mathbf{q}_{\alpha}, \mathbf{q}_{\gamma}\right) \Lambda_{\gamma n^{\prime \prime}, \beta n^{\prime}}\left(\mathbf{q}_{\gamma}, \mathbf{q}_{\beta}\right)
$$

where $\bar{\delta}_{\alpha \gamma}=1-\delta_{\alpha \gamma}$. An alternative procedure would be to solve for the eigenvalues and eigenfunctions of the overlap kernel, and to use its spectral decomposition to construct $\Lambda$. The latter procedure would be especially useful when the overlap operator $\Delta$ is singular (i.e., one or more eigenvalues are zero), in which case $\Lambda$ is to be taken as the generalized inverse. In either case, the projector is given as

$$
\Pi^{\mathrm{CRC}}=\sum_{\alpha=1}^{3} \sum_{n=1}^{N_{\alpha}} \sum_{\beta=1}^{3} \sum_{n^{\prime}=1}^{N_{\beta}} \int \mathrm{d} \mathbf{q}_{\alpha} \int \mathrm{d} \mathbf{q}_{\beta}\left|\phi_{\alpha n} \mathbf{q}_{\alpha}\right\rangle \Lambda_{\alpha n, \beta n^{\prime}}\left(\mathbf{q}_{\alpha}, \mathbf{q}_{\beta}\right)\left\langle\phi_{\beta n^{\prime}} \mathbf{q}_{\beta}\right|
$$

which can be written in abstract notation as $\Pi^{\mathrm{CRC}}=\sum_{\alpha} \sum_{\beta} \Pi_{\alpha} \Lambda_{\alpha \beta} \Pi_{\beta}$. Note that $\Pi_{\alpha} \Pi^{\mathrm{CRC}}=\Pi^{\mathrm{CRC}} \Pi_{\alpha}=\Pi_{\alpha}$.

\section{THE COUPLED-PSEUDO-REACTION-CHANNEL METHOD}

We now replace the exact Hamiltonian by its restriction $H^{\mathrm{CRC}}$ (三 $\Pi^{\mathrm{CRC}} H \Pi^{\mathrm{CRC}}$ ) on the approximation space, and look for the solutions of $\left(E-H^{\mathrm{CRC}}\right)\left|\Psi^{\mathrm{CRC}}\right\rangle=0$ in the time-independent treatment, or $\left(i \frac{\partial}{\partial t}-H^{\mathrm{CRC}}\right)\left|\Psi^{\mathrm{CRC}}\right\rangle=0$ in the time-dependent approach, subject to appropriate scattering boundary conditions, or a wave-packet initial condition, respectively. Concentrating on the stationary formulation, we expand the total wave function as

$$
\left|\Psi^{\mathrm{CRC}}\right\rangle=\sum_{\beta=1}^{3} \sum_{n=1}^{N_{\beta}}\left|\phi_{\beta n} f_{\beta n}\right\rangle=\sum_{\beta=1}^{3} \sum_{n=1}^{N_{\beta}} \int \mathrm{d} \mathbf{q}_{\beta}\left|\phi_{\beta n} \mathbf{q}_{\beta}\right\rangle f_{\beta n}\left(\mathbf{q}_{\beta}\right) .
$$

Requiring that the error $|\mathcal{E}\rangle\left(\equiv(E-H)\left|\Psi^{\mathrm{CRC}}\right\rangle\right)$ is orthogonal to the approximation space (i.e., $\Pi^{\mathrm{CRC}}|\mathcal{E}\rangle=0$, or, equivalently, $\Pi_{\gamma}|\mathcal{E}\rangle=0$ for $\left.\gamma=1,2,3\right)$, we obtain 


$$
\sum_{\beta=1}^{3} \sum_{n=1}^{N_{\beta}} \int \mathrm{d} \mathbf{q}_{\beta}\left[E \Delta_{\gamma n^{\prime}, \beta n}\left(\mathbf{q}_{\gamma}, \mathbf{q}_{\beta}\right)-H_{\gamma n^{\prime}, \beta n}\left(\mathbf{q}_{\gamma}, \mathbf{q}_{\beta}\right)\right] f_{\beta n}\left(\mathbf{q}_{\beta}\right)=0
$$

where $n^{\prime}=1, \ldots, N_{\gamma}, \gamma=1,2,3$, and $H_{\gamma n^{\prime}, \beta n}\left(\mathbf{q}_{\gamma}, \mathbf{q}_{\beta}\right)=\left\langle\phi_{\gamma n^{\prime}, \beta n} \mathbf{q}_{\gamma}|H| \phi_{\beta n} \mathbf{q}_{\beta}\right\rangle$.

Using $H=K_{\alpha}+h_{\alpha}+V^{\alpha}$, and $\left\langle\phi_{\alpha n}\left|h_{\alpha}\right| \phi_{\alpha n^{\prime}}\right\rangle=\delta_{n n^{\prime}} \tilde{\epsilon}_{\alpha n}$, we can rewrite (5) as

$$
\left(E-\tilde{\epsilon}_{\gamma n}-q_{\gamma}^{2} / 2 \nu_{\gamma}\right) f_{\gamma n}\left(\mathbf{q}_{\gamma}\right)=\sum_{\beta=1}^{3} \sum_{n^{\prime}=1}^{N_{\beta}} \int \mathrm{d} \mathbf{q}_{\beta}\left[\mathrm{V}_{\gamma n, \beta n^{\prime}}^{\text {post }}\left(\mathbf{q}_{\gamma}, \mathbf{q}_{\beta}\right)+\mathrm{V}_{\gamma n, \beta n^{\prime}}^{\mathrm{no}}\left(\mathbf{q}_{\gamma}, \mathbf{q}_{\beta}\right)\right] f_{\beta n^{\prime}}\left(\mathbf{q}_{\beta}\right)
$$

where

$$
\begin{aligned}
& \mathrm{V}_{\gamma n, \beta n^{\prime}}^{\text {post }}\left(\mathbf{q}_{\gamma}, \mathbf{q}_{\beta}\right)=\left\langle\phi_{\gamma n} \mathbf{q}_{\gamma}\left|V^{\gamma}\right| \phi_{\beta n^{\prime}} \mathbf{q}_{\beta}\right\rangle, \\
& \mathrm{V}_{\gamma n, \beta n^{\prime}}^{\text {no }}\left(\mathbf{q}_{\gamma}, \mathbf{q}_{\beta}\right)=\bar{\delta}_{\gamma \beta}\left\langle\phi_{\gamma n} \mathbf{q}_{\gamma}\left|\left(H_{\gamma}-E\right)\right| \phi_{\beta n^{\prime}} \mathbf{q}_{\beta}\right\rangle .
\end{aligned}
$$

In operator form, we have

$$
\left(E-\tilde{\epsilon}_{\gamma n}-K_{\gamma}\right)\left|f_{\gamma n}\right\rangle=\sum_{\beta=1}^{3} \sum_{n^{\prime}=1}^{N_{\gamma}}\left(\mathrm{V}_{\gamma n, \beta n^{\prime}}^{\text {post }}+\mathrm{V}_{\gamma n, \beta n^{\prime}}^{\text {no }}\right)\left|f_{\beta n^{\prime}}\right\rangle .
$$

Defining $\mathrm{G}_{\gamma n}^{0}\left(E^{+}\right)=\left(E+i \eta-\tilde{\epsilon}_{\gamma n}-K_{\gamma}\right)^{-1}$, and taking the initial state as $\left|\phi_{\alpha_{0} n_{0}} \mathbf{q}_{\alpha_{0}}\right\rangle$, with total energy $E_{\alpha_{0} n_{0} q_{0}}$, the solution of Eq. (8), subject to outgoing boundary conditions, satisfies

$$
\left|f_{\gamma n}\right\rangle=\delta_{\gamma \alpha_{0}} \delta_{n n_{0}}\left|\mathbf{q}_{\alpha_{0}}\right\rangle+\mathrm{G}_{\gamma n}^{0}\left(E_{\alpha_{0} n_{0} q_{0}}^{+}\right) \sum_{\beta=1}^{3} \sum_{n^{\prime}=1}^{N_{\beta}}\left(\mathrm{V}_{\gamma n, \beta n^{\prime}}^{\text {post }}+\mathrm{V}_{\gamma n, \beta n^{\prime}}^{\mathrm{no}}\right)\left|f_{\beta n^{\prime}}\right\rangle
$$

In matrix notation, we have $|\mathbf{f}\rangle=|\mathbf{i}\rangle+\mathbf{G}^{0} \mathbf{V}^{\mathrm{CRC}}|\mathbf{f}\rangle$, where $\mathbf{V}^{\mathrm{CRC}}=\mathbf{V}^{\text {post }}+\mathbf{V}^{\text {no }},|\mathbf{f}\rangle \equiv$ column $\left\{\left|f_{\gamma n}\right\rangle\right\},|\mathbf{i}\rangle \equiv$ column $\left\{\left|i_{\gamma n}\right\rangle\right\}$, with $\left|i_{\gamma n}\right\rangle=\delta_{\gamma \alpha_{o}} \delta_{n n_{o}}\left|\mathbf{q}_{\alpha_{o}}\right\rangle, n=1, \ldots, N_{\gamma}, \gamma=1,2,3$.

We now define the matrix $\mathbf{T}^{\mathrm{CRC}}$ of transition operators $\mathrm{T}_{\beta n, \alpha n^{\prime}}^{\mathrm{CRC}}$ via $\mathbf{T}^{\mathrm{CRC}}|\mathbf{i}\rangle \equiv \mathbf{V}^{\mathrm{CRC}}|\mathbf{f}\rangle$. In explicit notation, $\mathrm{T}_{\gamma n, \alpha_{0} n_{0}}^{\mathrm{CRC}}\left(E_{\alpha_{0} n_{0} q_{0}}^{+}\right)\left|\mathbf{q}_{\alpha_{0}}\right\rangle=\sum_{\beta=1}^{3} \sum_{n^{\prime}=1}^{N_{\beta}} \mathrm{V}_{\gamma n, \beta n^{\prime}}^{\mathrm{CRC}}\left|f_{\beta n^{\prime}}\right\rangle$. The transition operators satisfy

$$
\mathrm{T}_{\gamma n, \alpha_{0} n_{0}}^{\mathrm{CRC}}\left(\mathbf{q}_{\gamma}, \mathbf{q}_{\alpha_{0}} ; E_{\alpha_{0} n_{0} q_{0}}^{+}\right)=\mathrm{V}_{\gamma n, \alpha_{0} n_{0}}^{\mathrm{CRC}}\left(\mathbf{q}_{\gamma}, \mathbf{q}_{\alpha_{0}}\right)+\sum_{\beta=1}^{3} \sum_{n^{\prime}=1}^{N_{\beta}} \int \mathrm{d} \mathbf{q}_{\beta} \frac{\mathrm{V}_{\gamma n, \beta n^{\prime}}^{\mathrm{CRC}}\left(\mathbf{q}_{\gamma}, \mathbf{q}_{\beta}\right) \mathrm{T}_{\beta n^{\prime}, \alpha_{0} n_{0}}^{\mathrm{CRC}}\left(\mathbf{q}_{\beta}, \mathbf{q}_{\alpha_{0}} ; E_{\alpha_{0} n_{0} q_{0}}^{+}\right)}{E_{\alpha_{0} n_{0} q_{0}}+i \eta-\tilde{\epsilon}_{\beta n^{\prime}}-q_{\beta}^{2} / 2 \nu_{\beta}}
$$

These equations are of the standard (matrix-) LS form $\mathbf{T}^{\mathrm{CRC}}=\mathbf{V}^{\mathrm{CRC}}+\mathbf{V}^{\mathrm{CRC}} \mathbf{G}^{0} \mathbf{T}^{\mathrm{CRC}}$.

Using the integral formula $\left\langle\phi_{\gamma n} \mathbf{q}_{\gamma}\left|V^{\gamma}\right| \Psi^{\mathrm{CRC}}\right\rangle$ for rearrangement amplitudes for the transition $\left(\alpha_{0} n_{0}\right) \rightarrow(\gamma n)$, we can introduce another transition operator $\mathrm{U}_{\gamma n, \alpha_{0} n_{0}}^{(+) C R C}$ via $\mathrm{U}_{\gamma n, \alpha n_{0}}^{(+) \mathrm{CRC}_{0}}\left|\mathbf{q}_{\alpha_{0}}\right\rangle=\sum_{\beta=1}^{3} \sum_{n^{\prime}=1}^{N_{\beta}} \mathrm{V}_{\gamma n, \beta n^{\prime}}^{\text {post }}\left|f_{\beta n^{\prime}}\right\rangle$. In matrix notation, $U^{(+) C R C}=V^{\text {post }} \Omega^{\mathrm{CRC}}$, with $\Omega^{\mathrm{CRC}}(\equiv \mathbf{1}+$ $\mathbf{G}^{0} \mathbf{T}^{\mathrm{CRC}}$ ) being the CRC wave operator. The operators $\mathbf{U}^{(+) C R C}$ and $\mathbf{T}^{\mathrm{CRC}}$ are related by $\mathbf{U}^{(+) \mathrm{CRC}}=\mathbf{T}^{\mathrm{CRC}}-$ $\mathbf{V}^{\mathrm{no}} \boldsymbol{\Omega}^{\mathrm{CRC}}$. Since $\mathbf{V}_{\gamma n, \beta n^{\prime}}^{\mathrm{no}}\left(\mathbf{q}_{\gamma}, \mathbf{q}_{\beta}\right)$ vanishes for (physical) on-shell states $\left|\phi_{\gamma n} \mathbf{q}_{\gamma}\right\rangle$ with $n=1, \ldots, N_{\gamma}^{\text {bnd }}$, and $E=\epsilon_{\gamma n}+q_{\gamma}^{2} / 2 \nu$, the two operators are on-shell equivalent. We also note that $\mathbf{U}^{(+) C R C}$ is the solution of the integral equation $\mathbf{U}^{(+) C R C}=\mathbf{V}^{\text {post }}+\mathbf{U}^{(+){ }^{C R C}} \mathbf{G}^{0} \mathbf{V}^{\text {CRC }}$.

In some applications of the CRC method the nonorthogonality interaction $\mathbf{V}^{\mathrm{n}}$ is neglected. The corresponding approximate amplitudes $\tilde{\mathbf{T}}^{\text {post }}$ are then the solutions of $\tilde{\mathbf{T}}^{\text {post }}=\mathbf{V}^{\text {post }}+\mathbf{V}^{\text {post }} \mathbf{G}^{0} \tilde{\mathbf{T}}^{\text {post }}$.

\section{NUMERICAL RESULTS AND DISCUSSION}

The neglect-of-nonorthogonality approximation is tested on a model problem involving three identical particles which interact with separable $S$-wave pair potentials. This model, having both rearrangement and breakup channels, and being numerically solvable within the Faddeev formalism, provides a nontrivial test system for assessing the importance of nonorthogonality effects in the CRC approach. The pair potentials have the separable form $V_{\alpha}=\left|\chi_{\alpha}\right\rangle \lambda_{\alpha}\left\langle\chi_{\alpha}\right|$. We take $\chi(\mathbf{p})=\left(\beta^{2}+p^{2}\right)^{-1}$. Thus $V_{\alpha}, \alpha=1,2,3$, acts only on $s$ waves and support one bound state $\left(N_{\alpha}=1\right)$. The particle masses are taken equal to proton mass $M_{p}$, and we set $M_{p}=$ $\hbar=1$. We took $\beta=1.444 \mathrm{fm}^{-1}$, and $\lambda$ was chosen to give the bound-state energy of the two-nucleon system: $\epsilon=-0.0537 \mathrm{fm}^{-2}(\equiv 2.226 \mathrm{MeV})$. We further restrict our attention to zero total-angular-momentum state, so that the angular variables $\hat{\mathbf{p}}$ and $\hat{\mathbf{q}}$ disappear from Eq. (10). 
TABLE I. Comparison of exact, CRC and post-CRC results for the fermion and boson versions of the three-particle model.

\begin{tabular}{|c|c|c|c|c|c|}
\hline \multirow[b]{2}{*}{$E$} & \multicolumn{3}{|c|}{ Three-fermion case } & \multicolumn{2}{|c|}{ Three-boson case } \\
\hline & & $\operatorname{Re} T_{e l}^{\mathcal{A}}$ & $\operatorname{Im} T_{e l}^{\mathcal{A}}$ & $\operatorname{Re} T_{e l}^{S}$ & $\operatorname{Im} T_{e l}^{S}$ \\
\hline 0.1 & $\begin{array}{l}\text { Exact } \\
\text { CRC } \\
\text { Post }\end{array}$ & $\begin{array}{l}-0.0406 \\
-0.0404 \\
-1.7699\end{array}$ & $\begin{array}{l}-0.3122 \\
-0.3123 \\
-0.3944\end{array}$ & $\begin{array}{l}0.0059 \\
0.0137 \\
0.1208\end{array}$ & $\begin{array}{l}-0.0114 \\
-0.0097 \\
-0.0641\end{array}$ \\
\hline 0.3 & $\begin{array}{l}\text { Exact } \\
\text { CRC } \\
\text { Post }\end{array}$ & $\begin{array}{l}-0.1268 \\
-0.1270 \\
-0.0692\end{array}$ & $\begin{array}{l}-0.2393 \\
-0.2396 \\
-0.4090\end{array}$ & $\begin{array}{l}0.0911 \\
0.0987 \\
0.0628\end{array}$ & $\begin{array}{l}-0.0989 \\
-0.1007 \\
-0.1859\end{array}$ \\
\hline 0.5 & $\begin{array}{l}\text { Exact } \\
\text { CRC } \\
\text { Post }\end{array}$ & $\begin{array}{l}-0.1420 \\
-0.1425 \\
-0.0966\end{array}$ & $\begin{array}{l}-0.1775 \\
-0.1778 \\
-0.3982\end{array}$ & $\begin{array}{l}0.0880 \\
0.0862 \\
0.0243\end{array}$ & $\begin{array}{l}-0.1643 \\
-0.1672 \\
-0.1423\end{array}$ \\
\hline 0.7 & $\begin{array}{l}\text { Exact } \\
\text { CRC } \\
\text { Post }\end{array}$ & $\begin{array}{l}-0.1382 \\
-0.1388 \\
-0.0994\end{array}$ & $\begin{array}{l}-0.1347 \\
-0.1343 \\
-0.3136\end{array}$ & $\begin{array}{r}0.0562 \\
0.0538 \\
-0.0691\end{array}$ & $\begin{array}{l}-0.1944 \\
-0.1957 \\
-0.0815\end{array}$ \\
\hline 0.9 & $\begin{array}{l}\text { Exact } \\
\text { CRC } \\
\text { Post }\end{array}$ & $\begin{array}{l}-0.1282 \\
-0.1286 \\
-0.0928\end{array}$ & $\begin{array}{l}-0.1035 \\
-0.1034 \\
-0.2211\end{array}$ & $\begin{array}{r}0.0242 \\
0.0259 \\
-0.0674\end{array}$ & $\begin{array}{l}-0.2036 \\
-0.2044 \\
-0.0520\end{array}$ \\
\hline 1.1 & $\begin{array}{l}\text { Exact } \\
\text { CRC } \\
\text { Post }\end{array}$ & $\begin{array}{l}-0.1169 \\
-0.1178 \\
-0.1132\end{array}$ & $\begin{array}{l}-0.0813 \\
-0.0811 \\
-0.1827\end{array}$ & $\begin{array}{l}-0.0027 \\
-0.0045 \\
-0.0361\end{array}$ & $\begin{array}{l}-0.2011 \\
-0.2002 \\
-0.0243\end{array}$ \\
\hline 1.6 & $\begin{array}{l}\text { Exact } \\
\text { CRC }\end{array}$ & & & $\begin{array}{l}-0.0465 \\
-0.0506\end{array}$ & $\begin{array}{l}-0.1755 \\
-0.1725\end{array}$ \\
\hline 2.4 & $\begin{array}{l}\text { Exact } \\
\text { CRC }\end{array}$ & & & $\begin{array}{l}-0.0725 \\
-0.0672\end{array}$ & $\begin{array}{l}-0.1278 \\
-0.1279\end{array}$ \\
\hline
\end{tabular}

Two versions of this model were used: (i) three spinless bosons, and (ii) three spin- $\frac{1}{2}$ particles simulating the quartet spin state of the three-nucleon system. In terms of distinguishable-particle transition amplitudes, the symmetrized rearrangement amplitude for the boson case is given by $\mathrm{T}_{n n_{0}}^{\mathcal{S}}=\mathrm{T}_{1 n, 1 n_{0}}+\mathrm{T}_{2 n, 1 n_{0}}+\mathrm{T}_{3 n_{1}, 1 n_{0}}$, whereas the antisymmetrized rearrangement amplitude for the fermion case by $\mathrm{T}_{n n_{0}}^{\mathcal{A}}=\mathrm{T}_{1 n, 1 n_{0}}-0.5 \mathrm{~T}_{2 n, 1 n_{0}}-$ $0.5 \mathrm{~T}_{3 n, 1 n_{0}}$, with the coefficients of exchange amplitudes coming from the spin-isospin structure of the quartet state. Since there is one physical asymptotic state in each rearrangement, the physical rearrangement $T$-matrix elements (with $n=n_{o}=1$ ) are simply denoted as $\mathrm{T}_{e l}^{\mathcal{S}}$, or $\mathrm{T}_{e l}^{\mathcal{A}}$. The results labeled as exact in Table I were obtained by solving the Alt-Grassberger-Sandhas version of Faddeev equations ${ }^{12}$ with a Schwinger-type variational method. ${ }^{13}$ These reference solutions are stable to within $\mp 0.0005$ against the variations of the computational parameters (such as the type and number of basis functions, the number of quadrature points, etc.) of the solution method.

For the pseudostate CRC calculations, the two-particle basis set $\left\{u_{\alpha n}\left(p_{\alpha}\right)\right\}$ consisted of 15 associated Laguerre polynomials. The basis parameters are given in Ref. 11. For each rearrangement $\alpha$, the first 10 of the 15 pseudostates obtained from the diagonalization of $h_{\alpha}$ in the basis $\left\{u_{\alpha n}\right\}$ were used in the present calculations (i.e., $N_{\alpha}=10$ ). The $K$-matrix version of Eq.(10) was first regularized using a multichannel Kowalski-Noyes procedure, and the resulting set of integral equations were solved by quadrature discretization. The quality of results for $\mathrm{T}_{e l}$ were checked by also calculating the amplitudes $U_{e l}^{(+)}$via the integral formula. The calculated values for $T_{e l}$ and $U_{e l}^{(+)}$agreed to at least four places after the decimal point.

As the results in Table I show the post approximation is totally inadequate for the present models. Since there are three rearrangements in our model, and the approximation space used goes beyond the standard CRC space, this finding is perhaps not surprising. However, even at the $N_{\alpha}=1$ level (i.e., with the CRC expansion including only the proper rearrangement states), the full and post-approximation CRC equations yield different results. For example, at $E=1.1 \mathrm{fm}^{-2}$, such one-state calculations yield $\mathrm{T}_{e l}^{\mathrm{CRC}}=(-0.1111-i 0.2731)$, $\mathrm{T}_{e l}^{\text {post }}=(-0.0890-i 0.2911)$ for the boson model, and $\mathrm{T}_{e l}^{\mathrm{CRC}}=(-0.1561-i 0.1903), \mathrm{T}_{e l}^{\mathrm{post}}=(-0.0702-i 0.0163)$ for the fermion model.

It is noteworthy that the pseudostate-augmented CRC method (with the proper inclusion of nonorthogonality interaction) is capable of describing the effect of breakup channel on rearrangement amplitudes, even when the flux loss into the breakup channel is considerable. For instance, at $E=1.1 \mathrm{fm}^{-2}$, the total breakup probability (calculated from $1-\left|S_{e l}\right|^{2}$, with $S_{e l}=1-2 \pi \mathrm{T}_{e l}$ ) is $\sim 93 \%$ for the boson model, and $\sim 22 \%$ for the fermion model, and the CRC results for $\mathrm{T}_{e l}$ agree with the Faddeev results to within $\mp 0.002$. That is, the total breakup probability at a given collision energy is predicted quantitatively. 
At the highest energy considered, $E=2.4 \mathrm{fm}^{-2}$, there were 7 open pseudo-channels that effectively provided a sink for the total breakup flux. Although the rigorous breakup boundary conditions are violated in this approach, the mere presence of pseudoreaction channels seems to divert just the right amount of flux from reaction channels, and play much the same role as optical potentials do in the conventional applications of the CRC method. Why, and how, this happens is an open theoretical question. As mentioned in the Introduction, the use of pseudostates in the time-dependent CRC approach is a legitimate way of building a sufficiently large approx- imation space capable of describing the time-evolution of a three-particle wave packet. The connection between the time-dependent and time-independent versions of the pseudostate-augmented CRC method is currently under investigation.

\section{ACKNOWLEDGMENTS}

Part of this work was done while the author was visiting the University of New Mexico. The hospitality of Prof. C. Chandler and Prof. A.G. Gibson and the partial financial support through NSF Grant No. PHY-8802774 are gratefully acknowledged.
${ }^{1}$ T.Ohmura, B.Imanishi, M.Ichimura, and M.Kawai, Prog. Theor. Phys. 41, 391 (1969); T.Udagawa, H.H. Walter, and W.R. Coker, Phys. Rev. Lett. 31, 1507 (1973); Y.C. Tang, M. LeMere, and D.R. Thompson, Phys. Rep. 47, 167 (1978).

${ }^{2}$ S.R. Cotanch and C.M. Vincent, Phys. Rev. C 14, 1739 (1976).

${ }^{3}$ J.Z.H. Zhang and W.H. Miller, J. Chem. Phys. 91, 1528 (1989); Y. Sun, C.-h. Yu, D.J. Kouri, D.W. Schwenke, P. Halvick, M. Mladenovic, and D.G. Truhlar, J. Chem. Phys 91, 1643 (1989), and references cited therein.

${ }^{4}$ Z.C. Kuruoglu and F.S. Levin, Phys. Rev. Lett. 64, 1701 (1990).

${ }^{5}$ Z.C. Kuruoglu, Phys. Rev. A 42, 6314 (1990).

${ }^{6}$ C. Chandler and A. Gibson, J. Math. Phys. 14, 2336 (1977).
${ }^{7}$ Gy. Bencze, C. Chandler, and A.G. Gibson, Nucl. Phys. A390, 461 (1982).

${ }^{8}$ M.Birse and E.F. Redish, Nucl. Phys. A406, 149 (1983).

${ }^{9}$ A. Ben-Israel and T.N.E. Greville, Generalized Inverses: Theory and Applications (Wiley, New York, 1974).

${ }^{10}$ G. H. Rawitscher, Phys. Rev. 163, 1223 (1967); N. Austern, Phys. Rev 188, 1595 (1969); L.J.B. Goldfarb and K. Takeuchi, Nucl. Phys. A181, 609 (1971); T.Ohmura, B.Imanishi, M. Ichimura, and M. Kawai, Prog. Theor. Phys. 43, 347 (1970), and references cited therein.

${ }^{11}$ Z.C. Kuruoglu and F.S. Levin, Phys. Rev. Lett. 48, 899 (1982); Ann. Phys. (N.Y.) 163, 120 (1985).

${ }^{12}$ E.O.Alt, W.Grassberger, and W. Sandhas, Nucl. Phys. B2, 167 (1967).

${ }^{13}$ Z.C.Kuruoglu and D.A.Micha, J. Chem. Phys. 80, 4262 (1984). 